\title{
L’Autorité de la référence. Usages de la sharî̀a islamique dans le contexte judiciaire égyptien
}

\section{Baudouin Dupret}

\section{(2) OpenEdition}

1 Journals

Édition électronique

URL : http://journals.openedition.org/assr/1046

DOI : $10.4000 /$ assr. 1046

ISSN : $1777-5825$

Éditeur

Éditions de l'EHESS

\section{Édition imprimée}

Date de publication : 1 janvier 2004

Pagination : 189-210

ISBN : 2-222-96741-4

ISSN : 0335-5985

\section{Référence électronique}

Baudouin Dupret, «L'Autorité de la référence. Usages de la sharîa islamique dans le contexte judiciaire égyptien », Archives de sciences sociales des religions [En ligne], 125 | janvier - mars 2004, mis en ligne le 22 février 2007, consulté le 20 avril 2019. URL : http://journals.openedition.org/assr/1046 ; DOI : 10.4000 /assr. 1046 


\section{L'AUTORITÉ DE LA RÉFÉRENCE : USAGES DE LA SHAR $\hat{I}^{\prime} A$ ISLAMIQUE DANS LE CONTEXTE JUDICIAIRE ÉGYPTIEN (1)}

Pas plus la religion que le droit ne sont dotés d'une autorité intrinsèque, substantielle et naturelle. Autrement dit, l'autorité n'est pas une qualité inhérente aux objets, mais le fruit d'opérations continues d'imputation d'une capacité de peser sur le cours des choses. Il n'existe dès lors pas d'autorité d'un droit fondé religieusement dont on puisse parler en soi, mais seulement des situations ponctuelles où les gens s'orientent vers quelque chose qu'ils identifient comme étant du droit religieux et dont ils reconnaissent publiquement l'autorité, participant de ce fait aussi bien à la production de cette autorité qu'à sa réactualisation. On ne peut ainsi examiner la référence au droit islamique (sharî'a) en dehors des usages qui en sont faits de manière circonstancielle et située, en dehors des pratiques de référencement à un objet explicitement qualifié de juridiquement islamique dans des contextes variés, chacun soumis à ses contraintes propres. Au titre de ces cadres contextuels, l'enceinte judiciaire.

Affirmer que la recherche doit porter sur les pratiques contextualisées de référencement à un objet religieux revient à proposer une respécification de la question de l'autorité. Plutôt que de postuler l'existence d'une autorité religieuse, qu'elle soit textuelle ou institutionnelle, et se servir de ce concept comme d'une ressource permettant d'examiner les différents cas de figure d'un modèle sous-jacent, on suggère d'examiner la production contextuelle de l'autorité religieuse, c'est-à-dire de décrire comment les gens engagés dans des actions situées s'orientent vers un objet religieux et confèrent à sa référence une autorité manifeste et intelligible. En ce sens, l'autorité est un accomplissement et son caractère religieux vient de ce que les gens pointent explicitement vers cette dimension dans le cours de leurs interactions.

Cette contribution s'attachera à décrire dans le détail une séquence judiciaire portant, dans un cadre institutionnel égyptien, sur une question de statut personnel. On sait qu'il s'agit d'un domaine du droit traditionnellement présenté comme le

(1) Mes remerciements à Nathalie Bernard-Maugiron et Jean-Noël Ferrié, qui ont bien voulu lire une première version de ce texte et formuler des remarques toujours précises et pertinentes. 
dernier bastion de l'autorité du droit islamique en Égypte et dans le monde arabe. Le travail consistera à montrer les modalités par lesquelles cette autorité est produite, manifestée, référencée et organisée dans le contexte situé d'un procès en justice dans lequel une épouse demande au juge de prononcer le divorce en raison du préjudice dont elle souffre du fait de son mari qui a abandonné le domicile conjugal. Il s'agira donc de décrire comment se construit la pertinence de la référence à l'islam et au droit islamique. Ce faisant, on aura souligné à quel point l'autorité de la référence à la sharî'a est avant tout question de contexte institutionnel, de contrainte procédurale et de pertinence juridique, comment, en d'autres termes, elle s'inscrit dans l'accomplissement routinier de la profession de juge.

\section{La règle comme accomplissement pratique}

La question de l'autorité fait fréquemment l'objet d'un traitement, dans les travaux portant sur la norme dans l'espace arabo-musulman, que l'on pourrait qualifier d'essentialiste. À titre d'exemple, on peut citer deux ouvrages. D'une part, celui d'Oussama Arabi sur le droit islamique moderne (2). Il ressort clairement que, pour l'auteur, le droit islamique continue à déterminer le comportement des gens au quotidien. Pour Arabi, le droit islamique, loin d'être un simple corpus doctrinal, constitue une réalité vivante dans les sociétés musulmanes contemporaines où il est mis en œuvre comme droit positif de l'État. Sur la seule question sexuelle, l'auteur semble impliquer que les dispositions observables dans les traités de fiqh continuent d'exercer une influence sur le sens commun et sur la conception juridique d'aujourd'hui. Il s'agit là d'un postulat de continuité sociale, culturelle et historique. Empiriquement, toutefois, il semble bien difficile d'en faire la démonstration. L'auteur considère, en fait, que le sens commun envisage les rapports sexuels qui dépassent le flirt, s'ils ne sont pas validés par un contrat religieux, sous la catégorie du harâm. Cela montre, pour lui, que le sens commun musulman persiste à privilégier la forme contractuelle, ce qui nous renvoie, en d'autres termes, à l'encadrement de la sexualité par le droit religieux (3). La question est ici de savoir si l'on peut fonder sur l'observation du recours à une forme contractuelle l'affirmation d'une persistance de l'encadrement du social par la normativité religieuse. Si les gens sentent le besoin de recourir à une forme contractuelle minimale, il peut s'agir d'une question aussi bien de confort que d'habitude ou de conviction. Rien ne permet de trancher le débat dans le sens d'une internalisation des règles de la contractualisation matrimoniale renvoyant toutes à l'islam.

De manière autrement plus accentuée, Hocine Benkheira se fait le tenant d'une thèse considérant la religion comme une entité extérieure aux pratiques, non déterminée historiquement, sociologiquement, politiquement et culturellement, bref, un

(2) Oussama Arabi, Studies in Modern Islamic Law and Jurisprudence, La Haye-Londres-New York, Kluwer Law International, 2001.

(3) Sur ces questions, cf. Constant HAMÈs, « Quels rapports entre le droit religieux islamique et les normes et valeurs des sociétés ? L'exemple de la sexualité » in Jacques MaîTre, Guy Michelat, Religion et sexualité, Paris, L'Harmattan, 2003, pp. 141-150. 
système indépendant des sociétés et des individus qui les composent. Seraient opposés le système et le collectif, d'un côté, la pratique et l'individuel, de l'autre, avec en prime l'impossibilité de les réconcilier. Une fois écartées les pratiques parce qu'elles ne font pas système, sans doute - il devient possible à Benkheira d'affirmer la place centrale de la religion dans l'ensemble de l'aire islamisée, «car la religion, qui y joue un rôle moteur, pénètre l'ensemble du corps social, instille ses catégories et ses représentations dans le cœur des fidèles, voire même des membres non musulmans ou non croyants des sociétés, moule leur mode de pensée, leur imprime une disposition d'esprit, tend à uniformiser et à homogénéiser les discours ainsi que les comportements » (4). Il s'agit d'une autre forme de la théorie de l'incorporation (5) qui place la religion dans un domaine sui generis, celui qu'elle revendique, celui de l'exceptionnalité. Tout aussi exceptionnelle est alors la loi qui procède de la religion, la sharî̀ $a$ pour ce qui nous concerne. Celle-ci est érigée en " concept de toute société qui se développe dans le cadre de l'islâm », un objet «non empirique » (sic! Le concept n'est pas empirique dès lors qu'on ne s'intéresse pas aux pratiques du concept, dont on se demande toutefois quelles sont les manifestations en-dehors des pratiques) servant de pôle homogénéisateur du monde islamisé et de concaténateur des comportements du musulman. Le système normatif, dans sa genèse comme dans son évolution, serait donc antérieur aux individus qui s'y réfèrent. Autrement dit, il serait transcendant, l'indice de ceci étant que le système peut rendre compte des comportements alors que les comportements ne peuvent nullement rendre compte du système (comme si le système religieux n'était pas intégralement un fait pratique : énonciation de la règle, application, invocation, transgression, utilisation, manipulation, ignorance, etc.)

Face aux théories de l'incorporation, selon lesquelles les acteurs rencontrent des situations d'action auxquelles s'applique un ensemble de règles internalisées et agissent donc sous l'effet causal de l'autorité de ces règles, une approche par les pratiques montre, tout au contraire, l'impossibilité d'ouvrir la «boîte noire » de l'autorité de la règle en lui substituant une autre boîte noire, celle qui consiste à faire des gens les réceptacles individuels de principes culturels qui leur seraient en quelque sorte consubstantiels. Parler d'autorité, c'est, avant toute chose, s'interroger sur la question de savoir de l'autorité de quoi ou de qui il s'agit. L'autorité s'entend en effet comme la modalité d'une action (on agit avec autorité), la propriété d'une personne (on dit de quelqu'un qu'il a de l'autorité) ou la qualité propre à une norme (l'autorité de la règle). En ce sens, l'autorité apparaît toujours comme le prédicat de quelque chose qu'en quelque sorte elle accompagne. Dans le cas qui nous concerne, il s'agit donc de voir comment s'accomplit l'autorité qui s'attache, de manière redoublée (en droit et en religion), aux règles référées au corpus juridique islamique.

Traiter de l'autorité de la règle comme d'un prédicat ou d'une modalité s'attachant à celle-ci renvoie à la question de savoir ce qu'est suivre une règle. Wittgenstein montre qu'en théorie, toute règle peut faire l'objet d'un nombre

(4) Mohammed Hocine BENKHEIRA, Islâm et interdits alimentaires. Juguler l'animalité, Paris, PUF, 2000, p. 8 (coll. " Pratiques théoriques » [cf. Arch. 116.4]).

(5) Ceci n'a rien d'étonnant, dès lors qu'une filiation explicite aux travaux de Pierre Legendre est revendiquée, jusque dans le titre même de l'ouvrage précédent de l'auteur (Mohammed Hocine Benkheira, L'amour de la loi. Essai sur la normalité en Islâm, Paris, PUF, 1997, (coll. «Politique d'aujourd'hui »), [cf. Arch. 110.7]). 
indéfini d'interprétations. Ceci pose le problème de l'arrêt de l'indétermination. Il est absurde de penser qu'il faille connaître à l'avance l'infinité des lectures possibles d'une règle pour connaître la version autorisée (6). Contrairement à la tendance sceptique pour laquelle les actions ordonnées ne sont pas déterminées par des règles mais par des conventions sociales et des dispositions apprises qui bloquent la possibilité d'une régression interprétative (7), il convient d'adopter une perspective praxéologique pour laquelle les règles ne peuvent être séparées des conduites pratiques. Seule une lecture biaisée conduit à lire le paradoxe de Wittgenstein dans une perspective conventionnaliste (8).

Règles et conduites ne peuvent être traitées séparément. Autrement dit, la relation qui les unit ne procède pas de facteurs sociologiques extrinsèques tels que la convention d'une communauté. Le raisonnement de Wittgenstein vise à démontrer l'absurdité d'une représentation quasi-causale du fait de suivre une règle qui ferait de celle-ci une abstraction enclenchant des mécanismes mentaux, au lieu de montrer que le fait de suivre une règle repose sur un accomplissement pratique (9). La règle ne trouve son intelligibilité que dans l'adhésion pratique qui y est faite, c'est-à-dire dans l'ordre des activités concertées qui sont déjà en place quand une règle est énoncée, violée, ignorée ou suivie. L'énoncé de la règle est compris dans et à travers ces pratiques et il ne peut en aucune façon en être abstrait. Suivre une règle ne revient donc pas à l'interpréter, comme si sa signification était déjà pleinement contenue dans sa formulation abstraite, mais à agir et à rendre publique notre compréhension en agissant d'une manière qui manifeste notre accord avec elle. L'expression de cet accord permet en même temps de pointer les erreurs, les perturbations et les incompréhensions, toutes choses dont les auteurs sont comptables. Le sens de la règle n'est donc ni un objet trans-historique que les gens avaleraient et restitueraient de manière mécanique, ni le pur produit d'une convention sociale arrêtant le processus d'indétermination, mais une pratique qui en manifeste la compréhension (par mon action, je montre que j'ai compris) - et non pas l'interprétation (il n'est pas besoin d'une opération réflexive d'interprétation pour suivre la règle). Quant à l'autorité qui s'attache à la règle, elle ne procède pas de la crainte d'une sanction, de significations intangibles dont cette règle serait le condensé ou de la grandeur de ce à quoi elle réfère. Cette autorité renvoie au fait que, quand les gens suivent la règle, c'est de manière incontestée, routinière, non problématique, aveugle (au sens où elle ne fait pas l'objet d'une interrogation systématique quant à sa signification). En ce sens, c'est la pratique aussi qui fait l'autorité.

Si l'on en revient aux travaux d'Arabi et de Benkheira, on remarquera que, dans les deux cas, la règle est présentée comme indépendante de toute pratique. Elle existe, elle est dotée d'un sens clair et elle détermine le comportement des gens de manière univoque. Ainsi, les relations sexuelles hors-mariage sont-elles

(6) Charles TAYLOR, « Suivre une règle », Critique, août-septembre 1995, pp. 554-572.

(7) Saul Kripke, Wittgenstein on Rules and Private Language, Cambridge, Harvard University Press, 1982.

(8) Michael Lynch, Scientific Practice and Ordinary Action: Ethnomethodology and Social Studies of Science, Cambridge, Cambridge University Press, 1993 ; ID., « Après Wittgenstein. Le tournant de l'épistémologie à la sociologie des sciences », in Michel DE Fornel, Albert OGIEN, Louis QuÉRÉ, dirs., L'ethnométhodologie. Une sociologie radicale, Paris, La Découverte (colloque de Cerisy), 2001, pp. 123-160.

(9) Stuart G. Shanker, Wittgenstein and the Turning-Point in the Philosophy of Mathematics, Albany, State University of New York Press, 1987. 
rares, parce qu'elles ressortissent de la catégorie religieuse infâmante du harâm. Ainsi encore, tel interdit alimentaire s'impose-t-il nécessairement du fait même de son inscription dans la normativité islamique. À chaque fois, la règle est revêtue d'une autorité intrinsèque qui tient à son appartenance au registre du religieux. Cette propriété lui permet d'échapper à toute herméneutique. Si les normes juridiques ont, selon la formule de Herbert Hart, une « texture ouverte » (10), les normes religieuses sont, pour ces deux auteurs, des objets rigides et atemporels, à l'abri des aléas et de la contingence, en vertu même de leur inspiration divine. À l'inverse, notre position consiste à considérer la règle comme un accomplissement pratique et son autorité comme une modalité procédant de l'orientation publique des gens vers la force qu'ils lui reconnaissent. La règle ne préexiste donc pas à sa pratique et son autorité n'en est pas une qualité intrinsèque. Règle et pratique sont, au contraire, indissociables, et c'est de cette pratique même qu'émerge l'autorité de la règle.

\section{Qu'est-ce que le droit islamique ? (11)}

La règle est un accomplissement pratique et son autorité, une modalité contextuelle. En ce sens, dire d'une règle qu'elle est islamique et, partant, lui reconnaître une autorité spécifique est possible à la seule condition que cette qualification procède de la pratique contextualisée des acteurs sociaux, non des présupposés que l'analyste peut avoir sur la règle en raison de sa connaissance de la trajectoire généalogique de cette dernière. Ainsi en va-t-il du statut personnel dans les pays musulmans. Le postulat est souvent fait que le droit de la famille ou du statut personnel y constitue le dernier bastion du droit islamique, au point que l'on devrait parler de «droit islamique du statut personnel» tel qu'appliqué en Égypte et non de droit égyptien du statut personnel. Cette propension à déterminer dans quelle mesure telle ou telle part du droit est islamique et dans quelle mesure telle ou telle part du droit doit ou non être expliquée par quelque développement historique du droit islamique tend à imposer aux phénomènes et activités juridiques leur structure au lieu de chercher à découvrir comment ils opèrent. Ceci tient aux présupposés concernant le modèle général dont tel ou tel système juridique serait l'exemple. En opérant de la sorte, la recherche manque le phénomène qu'elle prétend documenter. Autrement dit, la caractérisation a priori du droit du statut personnel en Égypte comme «droit islamique » ne procure aucun accès à ce que les gens font dans le contexte judiciaire égyptien quand ils traitent de questions touchant au statut personnel, toutes choses qui ne peuvent être menées à bien qu'en décrivant les pratiques des gens en-dehors de tout cadre interprétatif préétabli.

Cette critique peut s'appliquer à l'essentiel de la littérature traitant du droit islamique dans les sociétés modernes. On citera simplement, à titre d'exemple, la notice sur le tashrî̀ signée par Aaron Layish et Ron Shaham dans la nouvelle

(10) Herbert L.A. Hart, The Concept of Law, Oxford, Oxford University Press, 1961 (traduction par M. van de Kerchove, Le concept de droit, Bruxelles, Publications des Facultés universitaires Saint-Louis, 1976).

(11) Pour une présentation détaillée de l'argument, cf. Baudouin DuprET, « What Is Islamic law : A Praxiological Re-Specification » (à paraître). 
édition de l'Encyclopédie de l'Islam (12) Se fondant sur l'étymologie du mot "tashrî"», qui renvoie à la Loi religieuse (sharî̀a), les auteurs passent totalement sous silence le fait que le terme sert aujourd'hui à désigner la notion de législation dans son acception la plus générale. À partir de leur connaissance préétablie de la trajectoire étymologique du vocable, ils imposent donc au phénomène législatif une nature qui ne peut en aucune façon être documentée. L'entreprise ne s'arrête pas là, toutefois. S'attelant à évaluer les transformations du tashrî dans le monde contemporain, Layish et Shaham établissent une distinction entre sharî'a orthodoxe et sharî'a déviante. Ils affirment ainsi que certains pays, comme l'Égypte et le Soudan, dans leur tentative de mettre en œuvre le droit islamique, ont tellement dévié de la sharî'a qu'ils ont fini par en provoquer la distorsion. Pareille affirmation n'est cependant pas sans poser un problème sociologique et épistémologique majeur. Elle suppose, en effet, que les chercheurs doivent juger de la normalité ou de la déviance de pratiques actuelles à l'aune de règles orthodoxes paradigmatiques, règles qu'il leur appartient d'identifier. Cela suppose aussi que les chercheurs sont autorisés à adopter une position ironique, surplombante, à l'égard des manières multiples qu'auraient les gens de se tromper eux-mêmes et d'être trompés par leurs gouvernements quand ils adhèrent à telle ou telle conception du droit islamique. En un mot, les chercheurs occuperaient une position leur permettant de savoir ce que les gens engagés dans une pratique quotidienne font mieux que ces gens eux-mêmes. Si ces derniers adoptent des législations déviantes, c'est par devers eux, inconsciemment. Enfin, cette affirmation suppose aussi que les catégories sont des réceptacles de significations qui traversent le temps et restent nécessairement pertinentes dans le contexte de leur usage présent. On pourrait toutefois faire remarquer que cette affirmation ne parvient, au mieux, qu'à montrer que les interprétations et pratiques juridiques ne demeurent pas constantes à travers l'histoire. Le gain de l'argument est faible - pour ne pas dire nul - et son coût élevé. Les sciences sociales et la philosophie ont depuis longtemps montré que les textes ne flottent pas dans le vide, qu'ils n'ont pas de signification en soi, qu'ils n'existent pas en dehors de l'action de les écrire et de les lire ; en un mot, qu'ils n'ont pas de sens littéral pur. Autrement dit, l'évaluation de pratiques contemporaines en termes d'orthodoxie et de déviance place le débat sur un terrain normatif tout en contournant la question centrale qui est de savoir ce que font les gens et comment ils le font quand ils font référence à la sharî'a aujourd'hui.

En général, caractériser le droit d'islamique renvoie simultanément à deux choses différentes : le droit islamique comme simple référence à l'islam dans un environnement juridique; et le droit islamique comme un système juridique qui peut être assimilé au corpus classique du fiqh. Dans ce dernier cas, il faut pouvoir disposer d'une définition substantielle du droit islamique dont les critères seraient satisfaits par le droit spécifique auquel on s'intéresse, de telle sorte qu'il puisse être tenu pour un exemple particulier du modèle général. Ceci soulève plusieurs questions : quels sont les critères constitutifs ? Y a-t-il un système juridique islamique paradigmatique qui puisse servir d'étalon d'évaluation d'autres instances du modèle ? De quoi ce système juridique idéal est-il le modèle ? Qui détient l'autorité permettant de faire de ce modèle le modèle faisant autorité ? La littérature sur la question passe par différentes positions. Il s'agit parfois de s'en tenir aux termes propres utilisés par les gens; parfois, il semble que les gens se mentent à

(12) Aaron Layish, Ron Shaham, «tashrî»», Encyclopédie de l'Islam, deuxième édition. 
eux-mêmes et ne disent pas ce qu'ils pensent ou encore que ce qu'ils pensent est faux. Considérons d'abord le fait de s'en tenir aux propos formulés par les gens. Par exemple, les gens disent que la condamnation de l'apostasie est un principe juridique islamique. Ceci devrait être considéré comme du droit islamique dès lors que les gens s'y réfèrent en tant que droit islamique. Une toute autre question est cependant de savoir si la condamnation de l'apostasie à laquelle ils se réfèrent est la condamnation de l'apostasie telle qu'organisée dans les traités de fiqh. On retrouve le vieux problème du nominalisme : l'usage du même mot à différentes époques ne signifie pas nécessairement que le mot renvoie à la même signification et à la même définition technique. On peut établir des connections, des histoires biographiques de concepts, etc., mais cela ne devrait pas conduire à présumer du caractère identique de deux ou plusieurs usages du même mot sur la seule base d'une forme linguistique. Deuxièmement, l'idée que les gens se mentent à eux-mêmes. Par exemple, l'affirmation que, bien que les juges égyptiens n'utilisent plus le mot «dhimma», le système de la dhimma est demeuré en place en droit égyptien (13). C'est un point de vue parfaitement ironique qui suppose que les chercheurs savent mieux que les gens engagés dans une pratique professionnelle, qu'ils occupent une place de surplomb permettant de dire ce qu'est la réalité, laquelle est différente de ce que les gens pensent. De plus, c'est une conception métaphysique et déterministe. Métaphysique, dès lors qu'elle affirme que les structures sont permanentes, même si les gens n'en connaissent plus rien. Les structures flottent dans l'histoire et les gens sont comme génétiquement enfermés dans ces structures et contraints par elles. Déterministe, au sens où les gens sont déterminés par des contraintes extérieures et ne produisent ni ne transforment quoi que ce soit, mais ne font que reproduire le passé. L'on présume donc, quand on prétend que les gens ne disent pas ce qu'ils pensent ou que ce qu'ils pensent est faux, que les chercheurs peuvent occuper cette position de surplomb qui les autorise à dire aux gens ce qui est vrai et faux, en dépit de ce qu'ils pensent, disent et font. Bien que ces deux positions soient contradictoires, leur combinaison rend l'argument infalsifiable, puisqu'il reste toujours possible de passer de l'un à l'autre en fonction de l'objection qui est formulée ou du problème auquel on est confronté.

Il convient donc de reformuler la question. Il n'y a en effet aucune raison de présumer que ce à quoi les gens se réfèrent comme étant du droit islamique est identique à cet ensemble de dispositions techniques formant le modèle idéalisé du droit islamique. Il n'y a pas non plus de raison de présumer le contraire. La question de savoir si ce à quoi les gens se réfèrent comme étant du droit islamique correspond ou non au modèle idéalisé du droit islamique n'est simplement pas pertinente. On ne peut y répondre, parce que la question est totalement désincarnée de pratiques réelles. De plus, cette question ne porte pas sur le phénomène qu'elle est censée traiter, à savoir la pratique consistant à faire référence au droit islamique. À la question : «Qu'est-ce que le droit islamique?», il faudrait substituer la question: «Que font les gens quand ils font référence au droit islamique?».

Traiter la question de l'autorité du droit islamique sous l'angle du déploiement contextuel de pratiques s'orientant vers ce droit, lui accolant une autorité spécifique et le qualifiant d'islamique, souligne l'impossibilité d'isoler une question des

(13) Maurits Berger, «Public Policy and Islamic Law: The Modern Dhimmî in Contemporary Egyptian Family Law », Islamic Law and Society, 8 (1), 2001, pp. 88-136. 
détails circonstanciels de son déploiement. Autrement dit, étudier le droit signifie nécessairement étudier le «droit en action » (14). Dans cette perspective, il y a peu de sens à faire du droit égyptien du statut personnel l'instance du modèle que serait le droit islamique. L'opération consistant à rattacher une instance à un modèle suppose un choix de caractéristiques que l'on suppose primordiales par rapport à d'autres et qui permettent de satisfaire les exigences d'identité et d'équivalence entre le cas concret étudié et l'abstraction formelle à laquelle il est censé appartenir. Ce choix consiste, par exemple, à identifier les caractéristiques du droit égyptien de la famille qui font que, tout comme le droit algérien de la famille et contrairement au droit danois de la famille, son appartenance au droit islamique de la famille puisse être attestée. Ceci se fait au prix d'une occultation massive de tout ce qui, dans ces mêmes droits, ne correspond pas aux caractéristiques communes à toutes les instances du modèle et sous couvert d'une relation purement nominale entre le modèle et ses instances. Avec pour résultat qu'on n'en sait à peine plus sur les phénomènes sous-jacents à ce jeu de catégorisations, l'objet de la recherche se voyant imposé par avance un format préconçu par le chercheur. En lieu et place de ce souci de qualifier quelque chose $d$ ' " islamique ", on suggère de centrer l'attention sur la question de comment les gens, dans des contextes variés, s'orientent vers quelque chose qu'ils appellent «droit islamique ». Cette attitude indifférente tourne le dos à toute approche fondationnaliste qui chercherait des moyens extrinsèques de mesure et d'intelligibilité et appelle à l'examen des méthodes utilisées par les gens engagés dans une activité qu'ils identifient comme juridique et islamique pour produire localement cette vérité et cette intelligibilité leur permettant de coopérer et d'interagir de manière largement ordonnée. Une première conséquence de cette reformulation de la question est de ne pas chercher à définir l'objet étudié autrement que comme ce que les gens identifient comme tel. Dans notre exemple du droit islamique, il faudrait donc se contenter de dire que «le droit islamique est ce à quoi les gens se réfèrent comme étant du droit islamique » et s'attacher exclusivement à observer et décrire comment les gens engagés dans une pratique juridique conduisent leurs activités et établissent de manière pratique ce qui compte pour eux comme juridiquement islamique. Une instance n'est jamais qu'une instance d'elle-même, avec cette conséquence qu'il n'y a plus à chercher le modèle dont l'objet d'étude serait une instance, mais seulement à éclairer les mécanismes sociaux qui ont fait que cet objet s'est produit comme il s'est produit.

\section{La pratique du droit : contexte institutionnel, contrainte procédurale et pertinence juridique}

Concepts et catégories juridiques ne prennent leur signification qu'à la condition de ne pas les abstraire du contexte dans lequel ils ont été formulés. Quand les gens agissent et parlent, ils le font en sorte d'être intelligibles, si bien que ce sont les conditions concrètes et pratiques de production de cette intelligibilité qu'il

(14) Max Travers, John F. Manzo, eds., Law in Action: Ethnomethodological and Conversation Analytic Approaches to Law, Aldershot, Dartmouth/Ashgate, 1997. 
convient d'étudier. Si l'on considère, par exemple, les attributs sociaux que les participants à l'interaction mettent en avant dans le discours, il faut constater que ceux-ci ne sont pas attachés invariablement à ceux à qui ils sont imputés, mais qu'ils dépendent de l'environnement particulier dans lequel le discours prend place et des activités discursives dans lesquelles sont engagés, dans l'«ici et maintenant » de leur discours, les gens qui parlent. John Gumperz parle d' « indicateurs de contextualisation » (contextualisation cues) pour indiquer ces aspects du contexte qui sont pertinents pour interpréter ce qu'un intervenant veut dire (15).

On peut parler d' " ordre situé » pour exprimer cette relation indémêlable entre contexte et interaction (16). L'ordonnancement social des choses est une réalisation locale, qui peut s'analyser aussi bien en termes de «travail» des participants visant à ce que leurs activités s'ajustent à l'environnement qu'en termes de transformation et réorganisation de l'environnement sous l'effet de ce travail. La relation entre contexte et interaction se manifeste donc à différents niveaux. Tout d'abord, les différents participants à une interaction manifestent le fait qu'ils s'orientent vers le contexte particulier dans lequel ils interagissent. Le fait, par exemple, qu'un accusé s'adresse au substitut en l'appelant par le terme de «bey» n'est pas la simple marque d'une déférence, c'est aussi le signe d'une reconnaissance du contexte judiciaire de l'interaction et l'expression empiriquement attestable de l'autorité d'une des parties en présence vers laquelle s'oriente l'autre. Ensuite, il est possible de dire que le faire et le dire sont aussi bien modelés par le contexte que rénovateurs de celui-ci. Modelés par le contexte, en ce sens que ce qui se dit et se fait est le résultat de la configuration de l'activité qui précède comme du cadre plus large dans lequel les gens reconnaissent que l'activité se situe. Ainsi, la réponse de l'accusé procède de la question du substitut qui la précède comme du cadre du bureau du parquet général auquel les parties se réfèrent explicitement. Le dire et le faire sont aussi rénovateurs du contexte, dans la mesure où ils sont le résultat de séquences antérieures et la base de séquences ultérieures, si bien qu'ils fonctionnent en sorte de maintenir, ajuster ou modifier le sens du contexte qui prévalait et vers lequel les participants à l'interaction s'orientaient et dirigeaient leurs actions (17). Dans un interrogatoire du parquet, par exemple, la question du substitut peut être formulée de telle sorte qu'elle semble impliquer une responsabilité de l'accusé. La réponse de ce dernier peut à son tour être formulée de manière à anticiper cette implication, à repositionner l'acte incriminé et à le faire apparaître comme un acte justifié, ce qui entraînera un réalignement du substitut au tour de parole suivant. L'action (de parole) et le contexte sont à ce point liés que, généralement, c'est dans le contexte lui-même que les participants à une interaction trouveront les ressources et les indications leur permettant de comprendre leur environnement et de formuler l'action qui convient par rapport à celui-ci. On parlera alors, à la suite de Melvin Pollner, de contexte qui s'explique lui-même, par quoi on vise ces façons par lesquelles certaines situations s'ordonnancent en sorte de

(15) John Gumperz, Discourse Strategies, Cambridge, Cambridge University Press, 1982, p. 162.

(16) Paul Ten Have, George Psathas, Situated Order: Studies in the Social Organization of Talk and Embodied Activities, Washington (DC), University Press of America, 1995.

(17) Paul Drew, John Heritage, " Analyzing talk at work: an introduction », in Paul Drew, John Heritage, eds., Talk at Work. Interaction in Institutional Settings, Cambridge, Cambridge University Press, 1992, p. 18. 
manifester la familiarité et la routine auxquelles il convient de s'attendre à cet endroit (18).

Si l'on se tourne plus spécifiquement vers le contexte institutionnel, on peut identifier trois caractéristiques principales. Premièrement, le discours y est informé par son orientation vers des objectifs largement prédéfinis par l'insertion dans ce contexte institutionnel. Les participants à l'interaction institutionnelle, qu'il s'agisse des professionnels ou des profanes, manifestent très généralement le fait que leur conduite est conçue et orientée vers l'accomplissement de tâches institutionnelles ou la réalisation d'objectifs caractéristiques de cette institution, même si cela ne suit aucun déterminisme, les orientations pouvant fluctuer en fonction des contingences ponctuelles et locales de l'interaction et de la définition du statut des participants. Deuxièmement, on remarque que l'interaction est soumise, en contexte institutionnel, à un certain nombre de contraintes procédant spécifiquement de ce contexte institutionnel et de sa fonctionnalité. Ces contraintes, par rapport auxquelles les participants modèlent leur conduite, peuvent même avoir une nature formelle, voire juridique, comme c'est le cas de la procédure dans le contexte institutionnel judiciaire. Troisièmement, le discours en contexte institutionnel est organisé dans des cadres et procédures inférencielles propres à ce contexte. On entend par là que les inférences et implications tirées par les participants à une interaction en contexte institutionnel auront tendance à suivre des schèmes largement définis par leur insertion dans des modes de raisonnement propres à l'institution en cause (19). Ces caractéristiques propres à l'interaction institutionnelle entraînent plusieurs conséquences importantes. Parmi d'autres, on relèvera le fait que le système des tours de parole est institutionnellement structuré et qu'il structure à son tour fortement de multiples aspects de la conduite des participants à l'interaction institutionnelle. On remarquera aussi que ces derniers organisent leur conduite de manière à manifester et réaliser son caractère institutionnel. Les ressources de l'action sont, pour leur part, plus limitées qu'en contexte ordinaire ; les options et opportunités d'action sont réduites et relativement spécifiques. Par ailleurs, les procédures sont définies de manière plus précise et leur non-respect est sanctionné de manière plus stricte, en sorte qu'on peut observer leur respect plus systématique. Les choix lexicaux des interactants se révèlent aussi étroitement tributaires du contexte institutionnel, de la même manière que l'allocation des tours de parole, ce qui traduit à la fois la sélection des actions pertinentes (contrainte procédurale) et leur insertion dans un cadre de pertinence linguistique (pertinence juridique). Les séquences sont donc façonnées substantiellement par leur contexte institutionnel, tant et si bien qu'elles en deviennent caractéristiques. On remarquera aussi l'existence de schèmes standards d'interaction institutionnelle et de pratiques professionnelles visant à la gestion des tâches propres à cette interaction. Ainsi, les professionnels affichent-ils généralement une position neutre, neutralité qu'ils produisent par, précisément, des choix lexicaux, procéduraux et séquentiels. Enfin, il convient d'indiquer que les interactions institutionnelles révèlent généralement une structure asymétrique, même si l'existence de ce type d'asymétries ne peut être postulée du seul fait que l'interaction se passe dans un cadre institutionnel.

(18) Melvin Pollner, «Explicative Transactions: Making and Managing Meaning In Traffic Court », in George Psathas, ed., Everyday Language: Studies in Ethnomethodology, New York, Irvington Publishers, 1979.

(19) Paul Drew, John Heritage, « Analyzing talk at work: an introduction », op. cit., pp. 22-23. 
On remarquera, en outre, que le professionnel engagé dans la routine de sa profession s'oriente très généralement vers la production publique du fait qu'il accomplit correctement son travail. Cela se traduit dans l'accomplissement d'une double correction procédurale, dans l'expression écrite de la conduite des différentes procédures, d'une part, dans la rédaction du jugement, de l'autre. L'affirmation que la procédure est importante en droit peut sembler triviale à un juriste. Ceci ne dispense toutefois pas de l'examen attentif de la façon qu'ont les gens de manifester la compréhension pratique qu'ils ont de cette importance. Cet examen doit nécessairement se faire par la description détaillée du détail praxéologique de la production et de la manifestation de la procédure en contexte. Ces contraintes procédurales, vers lesquelles les acteurs s'orientent explicitement, ne correspondent en effet pas à un ensemble de règles abstraites tirées d'un système juridique extérieur, historique et surplombant, mais elles correspondent à la performance routinière et bureaucratique des professions du droit. La plupart des documents contenus dans un dossier judiciaire traduisent l'orientation des juges, procureurs et autres professionnels vers cette correction procédurale. Ceci est directement lié à la séquence générale du jugement, dans laquelle les participants s'adressent à des gens qui ne sont pas nécessairement physiquement présents dans la pièce, mais forment une audience écoutant ou regardant, pour ainsi dire, par-dessus l'épaule et virtuellement susceptible d'invalider les procédures pour vice de forme.

À tout ceci vient enfin s'ajouter le fait que cette activité est menée à des fins spécifiques, ce qu'on appellera la production de la pertinence juridique. Les différentes situations factuelles particulières dont l'instance judiciaire est saisie n'attendent pas «en étant déjà distinguées les unes des autres et caractérisées comme les instances de la règle générale dont l'application est en cause, pas plus que la règle elle-même ne se manifeste pour revendiquer ses propres cas d'application»(20). Cela ne signifie pas que l'interprétation est une pure affaire de conventions sociales. Il existe aussi un "point de vue interne » qui fait que les gens suivent des règles par habitude, s'en servent comme base de leurs décisions ou encore s'y réfèrent comme à un modèle de comportement. De plus, pour un nombre limité de cas nécessitant une véritable interprétation de la règle applicable, il est une multitude de situations dans lesquelles les gens n'interprètent pas, mais suivent la règle, tout simplement, parce que sa signification ne pose aucun problème. En ce sens, Hart adopte une position proche de celle que nous avons décrite dans notre discussion des interprétations de Wittgenstein sur la question de suivre une règle. La texture ouverte du droit n'est donc pas une absence de toute texture, mais elle est aussi un cadre contraignant vers lequel s'orientent les praticiens. Si les faits ne sont jamais des faits bruts, le droit applicable est un objet (au moins potentiel) d'interprétation et la qualification des faits n'est pas une opération strictement objective, ce qui ne signifie pas que tout soit pure construction. Comme l'a montré Melvin Pollner, même si la déviance est créée par la qualification sociale d'un acte comme déviant, il faut bien constater que les membres de la société eux-mêmes se conçoivent comme confrontés à un ordre de choses dont la qualité déviante est présupposée indépendante de la réponse que lui apporte la société. Autrement dit, la règle et la déviance peuvent être considérées par les sociologues comme des

(20) Herbert L.A. HART, The Concept of Law, op.cit., p. 123. 
artéfacts, elles sont conçues et vécues par les acteurs sociaux comme des catégories signifiantes et objectives (21). Les gens ont donc clairement tendance à objectiver les faits et les catégories juridiques, catégories qui «constituent l'équipement conceptuel de base avec lequel des gens tels que les juges, les avocats, les officiers de police et les assistants de probation organisent leurs activités quotidiennes»(22). Tout ceci signifie que la seule identification des catégories juridiques ne peut suffire, mais qu'il convient toujours d'examiner et décrire comment les gens s'orientent pratiquement vers elles.

\section{Pratique judiciaire et référence textuelle en matière de statut personnel}

Les questions de statut personnel sont organisées, en Égypte, par une série de lois, essentiellement les lois $25 / 1920$ et $25 / 1929$, toutes deux amendées par les lois $100 / 1985$ et 1/2000. En l'absence de disposition législative explicite, la loi 1/2000 stipule que le juge doit s'en référer à « l'opinion qui domine dans l'école de l'imâm Abû Hanîfa ». En pratique, de nombreux juges font encore usage, en pareille situation d'incertitude, de la codification officieuse de Qadrî Pasha (1347 H.), qui est une compilation de dispositions inspirées de l'école hanafite. Depuis l'unification des cours et tribunaux égyptiens en un système national centralisé, le statut personnel est soumis à des règles de procédure communes à toutes les matières civiles et commerciales, quand du moins il n'existe pas de texte spécifique l'organisant (comme c'est le cas des dispositions de la loi 1/2000, par exemple). Les affaires sont examinées au premier degré par des tribunaux sommaires (mahkama juz'iyya) ou des tribunaux de première instance (mahkama ibtidâ'iyya kulliyya), en fonction de la nature du litige. Les tribunaux sont divisés en chambres, dont la chambre du statut personnel (dâ'ira al-ahwâl al-shakhsiyya) qui est compétente sur des questions financières (wilâya 'alâ al-mâl) et non financières (wilâya 'alâ al-nafs) relatives au statut personnel (mariage, divorce, héritage, filiation). Ceci est vrai du divorce judiciaire pour cause de préjudice, matière réglée à l'art. 6 de la loi 25/1929, telle qu'amendée en 1985, qui stipule: "Si l'épouse prétend que son époux l'a maltraitée de manière telle que cela rend impossible pour des gens de leur statut social de poursuivre leur relation maritale, elle peut demander au juge de les séparer, suite à quoi le juge lui accordera un divorce irrévocable s'il est établi que la conciliation est impossible entre eux. Si, toutefois, il [c'est-à-dire l'époux] refuse la demande et qu'elle répète sa plainte par la suite sans que le préjudice ne soit établi, le juge désignera deux arbitres et il statuera en vertu des dispositions des art. 7, 8, 9, 10 et 11 [de cette même loi] ».

Dès lors qu'une femme soumet au juge une requête lui demandant de prononcer un divorce pour cause de préjudice qu'elle subirait du chef de son mari, le travail du juge est contraint, au moins formellement, par les différents éléments

(21) Melvin Pollner, « Sociological and common-sense models of the labelling process », in Roy Turner, ed., Ethnomethodology, Harmondsworth, Penguin Books Ltd, 1974, p. 37.

(22) David Sudnow, « Normal Crimes », Social Problems, 12, 1965, pp. 251-276. 
de cette disposition législative. Un processus séquentiel est engagé au travers duquel le juge franchit une série d'étapes successives avant d'arriver à sa décision. Ceci apparaît dans la façon dont est charpenté le jugement.

\section{Tribunal de première instance, affaire No 858,1998 , statut personnel, Gîza}

«Au nom de Dieu, le Clément, le Miséricordieux » $\mathrm{Au}$ nom du peuple

Tribunal de première instance de Gîza pour le statut personnel - personnes / premier circuit shar ${ }_{\imath}$

Jugement

Lors de la session shar ${ }^{\imath}$ tenue publiquement au palais du tribunal le mardi 25/12/2000 m. (23)

Sous la présidence de Son Excellence M. [...], Président du tribunal

Et la participation de MM. [...] et [...], juges

En présence de M. [...], substitut du parquet

En présence de M. [...], greffier

Le jugement suivant a été rendu :

Dans la requête déposée par Mme [...]

\section{Contre}

M. $[\ldots]$

Enregistrée sur le rôle public au No. 858 de l'année 1998 m., S.P., plénier de Gîza

\section{Le tribunal}

Après audition de la plaidoirie, examen des documents et de l'opinion du parquet et la délibération en droit :

Attendu qu['il ressort des] faits de la cause que la demanderesse a déposé sa plainte en vertu du formulaire déposé au greffe du tribunal [...] et qu'elle a été signifiée légalement au défendeur, en conclusion de laquelle elle demande [que soit rendu] un jugement la divorçant, réclamant qu'il lui soit ordonné de ne plus s'opposer à elle dans ses affaires conjugales et le contraignant aux frais et dépens. [1]

En appui à cela, il est dit qu'elle est l'épouse du défendeur en vertu de [...] Celui-ci a cependant persisté à lui infliger de mauvais traitements [...] en se livrant à des voies de fait contre elles, en l'insultant et en délaissant le domicile conjugal [...] Elle lui a demandé le divorce [...] mais il n'a pas accepté. [...] [2]

Attendu que la requête a été examinée [...] Lors de la séance du [...], la demanderesse a comparu en personne avec un représentant (avocat) et il a présenté des pièces consistant en : [...]

1) L'original d'un certificat officiel [...] qui indique que la demanderesse est mariée au défendeur [...]

2) La photocopie de l'acte de mariage [...] Le tribunal a proposé [...] la conciliation qu'elle a refusée. [3]

À la séance $\mathrm{du}[\ldots]$ les deux parties à la cause ont été représentées [...] Celui qui a comparu pour la demanderesse a ajouté une nouvelle demande à la requête, à savoir [que soit prononcé] le divorce judiciaire (tatlîq) irrévocable (talaqa bâ'ina) de la demanderesse et du défendeur pour [cause de son] mariage à une autre [femme]. Le tribunal a ajourné l'examen de la requête [...] [4]

(23) La mention «m. » signifie « milâdî», c'est-à-dire « de l'ère chrétienne ». 
Lors de la séance du [...] la demanderesse fut représentée par son représentant (avocat). Il a présenté la requête [...] qui réclame en conclusion que [soit prononcé] un jugement [établissant] le divorce définitif pour [cause de] mariage avec une autre [femme] [...] Il a demandé que la requête soit réservée pour le jugement et le parquet a donné son avis au tribunal, qui a décidé lors de cette séance de soumettre la requête à investigation pour qu'en soient établis les éléments [...] [5]

En application de ce jugement, le tribunal a entendu les deux témoins de la demanderesse $[\ldots][6]$

Lors de la dernière séance des plaidoiries de conclusion, la demanderesse a comparu en personne, avec un représentant (avocat), et le tribunal lui a proposé la conciliation qu'elle a refusée. Le parquet a donné son avis au tribunal qui a fixé la requête pour jugement à la séance d'aujourd'hui. [7]

Le tribunal souligne, en exergue de son jugement, [... qu'il n'est pas] besoin de se prononcer sur l'objet de la demande additionnelle [8]

Attendu que [...] il est établi en vertu de l'article 6 du décret-loi No. 25/1929 m. que $[\ldots][9]$

L'article 6 n'a pas défini le [...] [10]

Le préjudice prévu par le texte de l'article 6 a pour condition : [...] [11]

Il est établi dans la jurisprudence de la cassation que [...] [12]

Le critère du préjudice au sens de l'article 6 de la loi 25/1929 m. est [...] [13]

Le préjudice est établi par le témoignage de [...] [14] $[\ldots][15]$

L'article $6[\ldots]$ ne dessine pas de voie précise pour la tentative de conciliation

Sur la base de ce qui vient d'être mentionné, la demanderesse a introduit sa requête $[\ldots]$. Le tribunal a entendu les deux témoins [...] Dès lors, les conditions de la demande en divorce judiciaire [...] étaient réunies [...] Il s'impose donc au tribunal de prononcer son divorce judiciaire d'avec le défendeur dans la forme d'un divorce irrévocable pour [cause de] préjudice. [16]

Attendu que, pour l'ensemble des dépens en honoraires des avocats [...] [17]

\section{Pour ces motifs}

Le tribunal décide du divorce judiciaire de la demanderesse [...] d'avec le défendeur [...] dans la forme d'un divorce irrévocable pour [cause de] préjudice, lui ordonne de ne plus s'opposer à elle dans ses affaires conjugales et le contraint aux dépens et à dix livres correspondant aux honoraires des avocats.

Le greffier

Le président du tribunal

La règle législative formulée à l'article 6 contraint le jugement en matière de divorce judiciaire pour cause de préjudice à suivre le schéma suivant : requête établissement du préjudice - tentative de réconciliation - jugement. Le texte du jugement révèle toutefois une structure interne légèrement différente, plus complexe et confuse, qui peut être schématisée comme suit :

- 1) introduction

- 2) requête [attendus 1 et 2]

o plainte [1]

o faits de la cause selon la demanderesse [2] 
- 3) procédure suivie par le tribunal [attendus 3 à 7]

o pièces présentées en appui à la requête + proposition de conciliation [3]

o audition des parties + demande additionnelle + ajournement [4]

o audition + requête de la demanderesse + demande de fixation [5]

o audition des témoins [6]

o dernières plaidoiries + proposition de conciliation + avis du parquet + fixation du jugement [7]

- 4) examen des moyens légaux [attendus 8 à 15]

o considération préliminaire (inutilité de la demande additionnelle) [8]

o concept de préjudice : absence de définition; conditions [9-11]

o concept de préjudice dans la jurisprudence de la Cour de cassation [12]

o critère du préjudice [13]

o témoignage en matière de statut personnel [14]

o tentative de conciliation [15]

- 5) application du droit aux faits de la cause [attendu 16]

- 6) dépens et demandes annexes [attendu 17]

- 7) sentence

Cette séquence, bien qu'enregistrée par le juge dans la forme d'un jugement, reflète la contrainte procédurale réelle sous laquelle il opère. Une de ses tâches principales, en tant que professionnel engagé de manière routinière dans son activité, est de manifester publiquement l'accomplissement correct de son travail. La production d'un jugement procéduralement impeccable relève de ces priorités, ce qui est montré publiquement dans le récapitulatif que le juge opère de toutes les étapes qui doivent être nécessairement franchies et qui ont été effectivement accomplies. À ce niveau procédural, il est clair que le juge s'oriente exclusivement vers les aspects techniques du droit égyptien de la procédure. Ceci peut inclure la référence à des dispositions explicitement reliées au droit hanafite, s'agissant des témoins par exemple (attendu 14), mais c'est, dans notre cas, une occurrence unique qui, de plus et surtout, survient par le truchement de la jurisprudence de la Cour de cassation. Autrement dit, même quand il y est fait référence, le droit islamique intervient par le truchement de l'intermédiation du droit positif et de ses institutions.

\section{Tribunal de première instance, affaire No 858, 1998, statut personnel, Gîza}

Le préjudice est établi par le témoignage de deux hommes ou d'un homme et de deux femmes, en vertu de l'opinion prépondérante de la doctrine d'Abû Hanîfa et en application de l'article $280 \mathrm{du}$ Règlement [d'organisation des tribunaux] de la sharî̀ $a$ (24) : "La preuve appartient à deux hommes ou bien un homme et deux femmes » (Cass. Civile, 28/2/1960 m., $11^{\mathrm{e}}$ année judiciaire, p.181).

La plupart des documents d'un dossier judiciaire montrent cette orientation des juges et autres professionnels vers la correction procédurale. Ceci tient à la

(24) La loi $1 / 2000$ reprend intégralement cette disposition. 
séquence générale du procès dans laquelle chaque participant s'adresse à tour de rôle à des gens qui, à un certain moment, ne sont pas présents physiquement dans la salle, mais constituent un auditeur silencieux vers qui les interactants se tournent au-delà de leurs échanges verbaux immédiats (25). La notion peut sans aucun doute être étendue aux personnes absentes auxquelles un document, tel que le jugement, est adressé. L'invalidation potentielle du jugement que pareille audience pourrait ultérieurement décider est directement prise en compte par les participants et se traduit dans l'attitude très conditionnée qu'ils adoptent à l'égard des procédures qu'ils sont requis de suivre. Ces contraintes procédurales ne sont pas considérées comme des éléments importés d'un quelconque système juridique externe, historique ou surplombant. Elles représentent plutôt les dimensions directes, évidentes, réelles et pratiques de la routine bureaucratique quotidienne de personnes engagées, dans l'Égypte d'aujourd'hui, dans différentes activités juridiques professionnelles.

À titre d'illustration, prenons au hasard un rapport de conciliation rédigé par le Comité d'arbitrage et de conciliation de Gîza dans une affaire de divorce unilatéral à la requête de l'épouse $(\mathrm{khul}$ '). Cette tentative de conciliation est imposée conformément à la nouvelle procédure mise en place par l'article 20 de la loi 1/2000. Comme on va le voir, ce document traduit la dimension avant tout bureaucratique et routinière de la pratique du statut personnel, alors même qu'il émane d'une institution explicitement religieuse, al-Azhar.

\section{Comité d'arbitrage et de conciliation, Rapport dans l'affaire No. 180/2000}

Au nom de Dieu le Clément, le Miséricordieux

al-Azhar al-sharîf

Objet : Rapport sur l'affaire No. 180

Département des recherches islamiques de l'année 2000 intentée par Mme [...]

Administration générale du prêche et de l'information religieuse

Zône de prêche de Gîza, Comité d'arbitrage et de conciliation

contre $[\ldots]$

\section{Rapport}

Son Excellence M. le Conseiller [...], Tribunal de Gîza-Nord, Premier circuit shar `̂, plénier, Nord

La paix soit sur vous, la clémence de Dieu et Sa bénédiction

L'honorable tribunal nous a désignés pour arbitrer dans la requête No. 180 de l'année 2000 intentée par Mme $[\ldots]$ contre $[\ldots]$

\section{Exécution du mandat}

1- L'épouse a comparu au bureau d'arbitrage et de conciliation de la région de prêche de Gîza et on a procédé à l'enregistrement de ses propos par écrit et oralement et à son audition. On a tenu plusieurs séances à son sujet. Il en ressort l'impossibilité d'un accord et d'une conciliation entre elle et son époux, sachant que l'époux n'a pas comparu en dépit de ce qu'il avait connaissance de ces séances. L'épouse a rapporté par ses propos qu'elle avait fait face à l'humiliation, à la diffamation, à l'insulte et à l'atteinte à son honneur de la part de l'époux mentionné. Il a ainsi prononcé des propos calomniateurs, ce qui a conduit à éprouver les enfants psychologiquement et à ce qu'elle le déteste et veuille en divorcer par voie unilatérale (mukhâla 'a) pour préserver

(25) Paul Drew parle d' « over-hearing audience ». Paul DrEw, « Contested Evidence in Courtroom Cross-Examination: The Case of a Trial for Rape », in Paul Drew, John Heritage, eds., Talk at Work. Interaction in Institutional Settings, op. cit. 
l'avenir des enfants. Elle a dit: Je crains de ne pas respecter les limites de Dieu avec lui (allâ uqîm hudûd allâhi ma'ahu).

2- On a procédé à l'envoi de télégrammes à l'époux et il n'a pas comparu dans les locaux du Comité d'arbitrage et de conciliation de la zône de prêche de Gîza jusqu'à la rédaction de ce rapport.

\section{Opinion des deux arbitres}

Après examen des pièces de l'affaire, des documents qui l'accompagnent, des propos de l'épouse et ce qui est demandé de l'époux, l'impossibilité de la coexistence et de la vie maritale entre eux est apparue manifeste. Nous portons ceci à [la connaissance de] votre Excellence. Il appartient à la justice du tribunal de juger de la manière qu'il jugera conforme.

La paix soit sur vous, la clémence de Dieu et Sa bénédiction

Premier arbitre

Deuxième arbitre

Outre la tentative de conciliation, dont ce rapport est censé attester, la structure du document montre au moins deux choses :

1) Le rapport est un accomplissement en soi. Dans ce rapport, les arbitres produisent toutes les caractéristiques qui manifestent le fait qu'ils agissent ès qualité et qu'ils maîtrisent les aspects techniques procéduraux rendant possible la production d'un rapport du bureau ad hoc d'al-Azhar.

2) Ce rapport fait partie d'une procédure globale. Il mentionne qu'il s'intègre dans une procédure plus englobante suivie dans le jugement d'une affaire qui a été transmise aux arbitres par le tribunal, en leur demandant de fournir une prestation exigée par la loi.

Parallèlement à ce souci de correction formelle, les professionnels engagés dans un procès judiciaire s'orientent vers la catégorisation juridique des faits dont ils sont saisis, ce qu'on pourrait appeler la production de la pertinence juridique. Dans le cas du divorce judiciaire pour cause de préjudice, doivent ainsi être résolues les questions de savoir ce qui a la qualité de préjudice et ce qui peut être tenu pour la cause de ce préjudice. L'article 6 de la loi de 1929 donne une définition très large du préjudice. Il est question de l'épouse alléguant que son mari lui fait subir des mauvais traitements tels que la poursuite de la relation conjugale est devenue impossible pour des gens de leur statut social. C'est donc au juge qu'il appartient de qualifier les faits de la cause de manière à pouvoir les faire entrer sous la définition de la loi. À cette occasion, il manifeste la contrainte qu'exercent sur lui les définitions données par la Cour de cassation, comme cela apparaît explicitement à la lecture de l'extrait suivant du jugement (attendus 12-15).

\section{Tribunal de première instance, affaire No 858, 1998, statut personnel, Gîza}

Il est établi dans la jurisprudence de la cassation que «le préjudice commis par l'époux est soit positif soit négatif. Le préjudice positif consiste dans le mal fait par l'époux à son épouse par [la voie de] coups et insultes que n'autorise pas la sharî a islamique et dont souffre l'épouse. Quant au préjudice négatif, cela consiste dans l'abandon par l'époux de son épouse. C'est le préjudice le plus grave, le préjudice qui déshonore l'épouse. Il suffit qu'il survienne du fait de l'époux contre l'épouse une 
seule fois pour qu'elle ait le droit de demander le divorce pour préjudice (Cass., 31/3/1984 m., p. 287, Majallat al-qadâ', 1984 m.).

Le critère du préjudice au sens de l'article 6 de la loi 25/1929 m. est personnel, non pas matériel, et son évaluation est ce qui rend la poursuite de la vie maritale impossible. [Son critère] est objectif, laissé au juge du fond, et il varie en fonction de l'environnement des deux époux, de leur niveau de culture et de leur milieu social (Cass., 1/11/1978 m., p. 1174).

\section{$[\ldots ;$ cité supra $]$}

L'article 6, bien qu'il conditionne le divorce judiciaire pour préjudice à l'incapacité du juge de concilier les époux, ne dessine pas de voie précise pour la tentative de conciliation entre les deux époux. Le tribunal a dès lors proposé la conciliation aux deux parties. L'époux a accepté et l'épouse l'a refusée. Ceci est considéré comme [l'expression de] l'incapacité du tribunal de concilier les deux époux comme l'exige l'article 6 (Cass., 14/47, $30^{\mathrm{e}}$ année judiciaire [sic !], p. 906).

Il est clair que la définition donnée par la Cour de cassation n'épuise pas l'incertitude à laquelle le juge est confronté quand il qualifie les faits. Cela ne signifie pas, pour autant, que le travail du juge est particulièrement compliqué ou arbitraire. Les catégories auxquelles le juge fait référence ont, pour lui, une nature objective, même si, comme on l'a vu, c'est sa propre qualification qui les objective. De plus, le processus juridique de qualification est totalement soutenu par un processus social de normalisation, c'est-à-dire par cet ensemble d'opérations par lesquelles le juge sélectionne de manière routinière certaines des caractéristiques d'un cas semblable à un type de cas commun, normal, usuel (26). C'est à ces catégories «normales ", qui sont dotées, au-delà de leur définition juridique, d'une dimension propre au sens commun, que le juge, tout comme le procureur, l'avocat, la victime, l'accusé, les témoins ou les experts, s'orientent.

Dans l'affaire déjà évoquée, l'épouse a mobilisé deux moyens successifs pour donner substance à la catégorie de préjudice : 1) l'abandon du domicile conjugal ; 2) l'existence d'un second mariage. Ni l'un ni l'autre ne sont toutefois explicitement mentionnés par la loi comme causes de préjudice. C'est donc le propre du travail du juge que de considérer que ces faits entrent dans la catégorie légale et, partant, sont susceptibles des conséquences juridiques attachées à cette qualification. Et, dans son travail, c'est à la seule référence législative et jurisprudentielle que le juge renvoie. L'autorité sur laquelle il se fonde dans l'accomplissement pratique de son travail est donc strictement référentielle. Elle est en outre positive (le législateur égyptien et le juge de cassation). Elle est enfin routinière, puisqu'elle procède à la répétition d'un schème procédural identifiable dans la plupart des affaires de divorce pour cause de préjudice (27).

Un certain nombre d'éléments concourant à l'élaboration de ce document textuel désengagé qu'est le jugement peuvent être identifiés grâce à l'examen minutieux des différentes étapes et procédures ayant soutenu le travail du juge (bien que

(26) David Sudnow, « Normal Crimes », art. cité.

(27) Cf. Baudouin Dupret, « The Practice of Judging: The Egyptian Judiciary at Work in a Personal Status Case ", in Muhammad Khalid Masud, Ruud Peters, David Powers, eds., The Application of Islamic Law in Courts, Leyde, Brill (à paraître). Cf. également Baudouin Dupret, Morale du jugement, jugement de la morale. Une étude praxéologique de la justice égyptienne et de son traitement de questions touchant à la moralité publique, Mémoire pour l'habilitation à diriger des recherches, Paris, IEP, 2003. 
ces étapes et procédures débouchent elles-mêmes sur la production de documents textuels en partie désengagés). Dans notre cas, c'est à la seule technique probatoire du témoignage qu'il est fait recours pour établir le type de dommage ayant débouché sur la survenance d'un préjudice. Faute de disposer du matériau pertinent dans cette affaire, on prendra l'exemple d'un témoignage rendu dans un cas de même type.

\section{Tribunal de première instance, affaire No 701, 1983, statut personnel, Gîza}

Le tribunal a appelé le premier témoin de la demanderesse et il a dit:

1- Je m'appelle [...] serment

2- Question du tribunal: Quelle relation as-tu avec les deux parties

3- Réponse du témoin : Mon lieu de travail est proche du bureau de poste où travaille la demanderesse

4- Q : De quoi témoignes-tu

5- R : La demanderesse est la femme du défendeur en vertu d'un contrat de mariage légal il y avait des disputes entre eux et j'ai vu l'époux de la demanderesse que je connais bien que je ne connais pas le lieu de sa résidence il lui lançait des paroles devant le bureau de poste dans lequel elle travaille l'appelant je l'ai entendu lui adresser la parole par t'es une pute t'es dégueulasse et d'autres mots du genre pendant près de deux ans et il y a un mois il a appelé la police contre elle parce qu'il y avait eu entre eux quelque chose que je n'sais pas

6- Q : Depuis combien de temps connais-tu l'époux de la demanderesse

7- $\mathrm{R}$ : Depuis près de deux ans

8- Q : Habite-t-il dans le quartier

9- $\mathrm{R}:$ Je n'sais pas

10- Q : Depuis combien de temps le défendeur lance des sales mots à la demanderesse

11- $\mathrm{R}$ : Depuis près de deux ans

12- Q : Quels sont les mots qu'il lui a lancés

13- R : Il lui a dit t'es une pute t'es dégueulasse et des mots de ce genre et c'était devant le bureau de poste

14- Q : La demanderesse a-t-elle souffert d'un préjudice à cause de ça

15- R : Oui elle s'est effondrée pendant qu'elle travaillait au bureau de poste

16- Q : Quelque chose d'autre à dire

17- R : Non

En dépit du fait que les témoignages soient supposés être retranscrits dans les propres mots des témoins, il est clair qu'ils sont reformulés, au moins partiellement, par le juge et son secrétaire. C'est pour cela qu'il est fait état de ce que le témoin a commencé son témoignage par l'établissement de ce que la demanderesse et le défendeur sont époux «en vertu d'un contrat de mariage légal». Outre ce processus de réécriture et d'édition, la nature largement stéréotypée de l'organisation du témoignage et la nature pré-allouée des tours de parole dans la production du témoignage méritent l'attention. Ces deux éléments tiennent au contexte institutionnel dans lequel ces témoignages sont déposés. De plus, tout ce qui est fait dans ce contexte est nécessairement géré par les participants à l'intérieur même du cadre 
contraignant de l'organisation pré-allouée de tours de parole. Autrement dit, dans le contexte judiciaire, contrairement à la conversation ordinaire, l'ordre des tours, de même que le type de chaque tour, est fixé. À l'intérieur de ce système allouant les tours de parole, aussi bien le juge que les témoins sont orientés vers la production d'une information qui puisse être juridiquement pertinente et vers la crédibilité de cette information. Du côté du juge, la crédibilité de l'information fournie par chaque témoin est testée par des questions touchant à la crédibilité du témoin lui-même. C'est pour cela que l'interrogatoire commence par une question portant sur « la relation avec les deux parties » du témoin (tour de parole 3 ). Cette crédibilité peut être aussi examinée en demandant au témoin de produire un compte rendu de première main de son témoignage (tour de parole 5) et en évaluant la crédibilité de ce récit global au moyen de questions demandant au témoin de confirmer ce qu'il a affirmé point par point (tours de parole 7-14). Il est par ailleurs clair que le juge cherche aussi à extraire certains éléments d'information - dimension temporelle du comportement (combien de temps ? : tour de parole 11), contenu exact du comportement (mots exacts utilisés par le mari : tours de parole 13 et 16), responsabilité (qui l'a fait?: tour de parole 11), nature du préjudice causé par le comportement (quel effet sur l'épouse ? : tour de parole 15) - qui forment les éléments constitutifs de la catégorie juridique de préjudice. Mises ensemble, les pièces détachées de cette recherche d'information concordent avec les éléments qui conditionnent la notion de préjudice tel que défini par le jugement et conformément à la jurisprudence de la Cour de cassation : «le mal fait par l'époux à son épouse sous forme de parole ou d'acte, ou des deux, d'une manière qui ne soit pas acceptable pour des gens de même statut, et [qui] constitue quelque chose de honteux et de dommageable qui ne puisse pas être supporté ».

\section{Une référence islamique occasionnelle et routinisée}

À l'endroit même où elle est supposée massive et écrasante, à savoir le droit du statut personnel, la référence à l'autorité du droit islamique est loin d'apparaître systématiquement. Certes, des mentions formelles de l'autorité de la religion figurent en bonne place. Ainsi, le jugement est-il rendu « au nom de Dieu ». Il l'est toutefois également « au nom du peuple », ce qui n'a jusqu'à présent inspiré aucun argument en faveur de l'autorité de ce dernier. Plus sérieusement, on remarque que cette question ne procède, dans le domaine du droit, ni du poids que la tradition scientifique semble vouloir imputer à la référence islamique ni des voies exceptionnelles que son expression est présumée suivre. En d'autres termes, la référence explicite à l'autorité de l'islam est occasionnelle.

Cette référence est, de plus, toujours médiatisée par le recours aux sources premières - parce que tenues pour telles dans les cursus d'enseignement des matières juridiques - du droit égyptien d'aujourd'hui : la loi et la jurisprudence. Elle s'inscrit donc dans la banalité et la routine de l'accomplissement pratique de l'activité du juge qui consiste, avant toute autre chose, à qualifier juridiquement des faits qui lui sont soumis. Ce faisant, le juge s'attache sans doute plus à manifester sa capacité à juger correctement, selon les standards de la profession, les 
contraintes formelles qui s'appliquent à son exercice, les sources juridiques sur lesquelles elle s'appuie et les normes du travail interprétatif qu'elle suppose, qu'à réitérer le primat islamique du droit qu'il met en œuvre. Il ne fait pas de doute que, si on interrogeait ledit juge, il ne manquerait pas de souligner la conformité de son activité et du droit qu'il applique à la norme religieuse islamique. Cette position ne relèverait, pour autant, que de l'ordre rétrospectif, a posteriori, de la justification. Dans le cours de son travail, le juge ne semble en revanche pas s'orienter massivement vers la nécessité d'évaluer le prédicat islamique de toute chose, même dans le domaine du droit où la généalogie islamique des règles semble la plus manifeste.

Faut-il en conclure à l'inexistence de l'autorité islamique de la règle en matière de statut personnel ? Conformément à ce qu'on énonçait en introduction, c'est davantage à une respécification de la question qu'on voudrait inviter. Au lieu de se demander de quel point de vue il faut se placer pour pouvoir évaluer l'autorité islamique de la règle, il convient d'observer, en contexte, comment les juges s'appliquent à mettre en œuvre les règles, à les référencer à des sources faisant autorité dans l'exercice de leur profession et à s'inscrire dans le cours banal et routinier de leur travail de juges. Il ne s'agit plus, alors, de s'interroger, à partir du droit, sur le concept d'autorité en islam, mais bien de décrire les modes d'usage et de référence à la règle de droit et la production toujours située et ponctuelle de son autorité. La règle de droit, de ressource explicative de la question plus vaste de l'autorité dans une religion, se trouve ramenée au statut d'objet de la recherche en tant que tel, son autorité n'étant plus que la traduction routinière, peu ou pas réflexive et donc quasiment aveugle de ce que cette référence normative s'impose au titre de l'accomplissement d'une activité professionnelle.

Baudouin DUPRET Institut Français du Proche-Orient CNRS - Damas 
Résumé

Il n'y a pas de question abstraite d'autorité en islam ou en droit islamique, mais uniquement des pratiques de référencement à un objet religieux dans des contextes variés, chacun soumis à des contraintes propres. Plutôt que de postuler l'existence d'une autorité religieuse, qu'elle soit textuelle ou institutionnelle, il convient d'observer comment les gens engagés dans des actions situées s'orientent vers un objet religieux et confèrent à sa référence une autorité manifeste et intelligible. En ce sens, l'autorité est un accomplissement et son caractère religieux vient de ce que les gens pointent explicitement vers cette dimension dans le cours de leurs interactions. À partir de la description détaillée d'une séquence judiciaire portant, dans un cadre institutionnel égyptien, sur une question de statut personnel, on montrera comment se construit la pertinence de la référence à l'islam et au droit islamique. Ce faisant, on aura souligné à quel point l'autorité de la référence à la sharî̀a est question de correction procédurale et de pertinence juridique et non d'ontologie religieuse.

\section{Abstract}

The issue of authority in Islam and Islamic law does not exist in abstracto, but only in practices of referring to some religious object in various contexts, each one being submitted to its own constraints. Instead of assuming the existence of religious authority, be it textual or institutional, it is suggested to observe how people engaged in situated actions orient themselves toward a religious object and give to its reference a manifest and intelligible authority. It means that authority is an accomplishment and its religious character proceeds from the people's explicit orientation to this dimension in the course of their interactions. Drawing from the detailed description of a judicial sequence, in an Egyptian institutional setting and on a personal-status case, this article will show how the relevance of the referring to Islam and Islamic law is built. By so doing, it will be stressed that the authority of this referring to sharîa is an issue of procedural correctness and legal relevance, not of religious ontology.

\section{Resumen}

No existen cuestiones abstractas de autoridad en el Islam o en el derecho islámico, sino sólo prácticas de referencia a un objeto religioso en contextos variados, cada uno de ellos sometido a sus propias tensiones. Más que postular la existencia de una autoridad religiosa, sea ésta textual o institucional, conviene observar cómo las personas comprometidas en acciones situadas se orientan hacia un objeto religioso y le dan a la referencia de éste una autoridad manifiesta e inteligible. En este sentido, la autoridad se cumple, y su carácter religioso viene de lo que las personas dirigen explícitamente hacia esta dimensión en el curso de sus interacciones. A partir de la descripción detallada de una secuencia judicial sobre una cuestión de carácter personal en el marco institucional egipcio, se mostrará aquí cómo se construye la pertinencia de la referencia al Islam y al derecho islámico. Así, habremos destacado hasta que punto la autoridad de la referencia a la sharî̀a es una cuestión de procedimientos correctos y de pertinencia jurídica, y no de ontología religiosa. 
Dépôt légal $-1^{\text {er }}$ trimestre 2004

Commission paritaire 1007 B 06233 



\title{
BULLETIN D'ABONNEMENT
}

\author{
À retourner à votre librairie ou aux \\ PRESSES UNIVERSITAIRES DE FRANCE \\ Département des Revues \\ 6, avenue Reille \\ 75014 PARIS \\ Téléphone : (33) 01.58.10.31.61 \\ Télécopie : (33) 01.58.10.31.82 \\ E.mail : revues@puf.com. \\ www.puf.com
}

Je souscris un abonnement pour 2004 à la revue :

Archives de sciences sociales des religions

Tarifs $2004:$ France $€ 72$ - Étranger $€ 86$ - Vente au numéro : $€ 22$

NOM

PRÉNOM

Adresse

Code postal

Ville

Je vous adresse ci-joint le montant de cette commande par :

( ) Chèque bancaire ou postal libellé à l'ordre des Presses Universitaires de France

( ) Virement postal sur le CCP 39233 A Paris

( ) Carte bancaire (Visa, Eurocard ou Mastercard, Amex, JCB, Diners-Club) $\mathrm{n}^{\circ}$

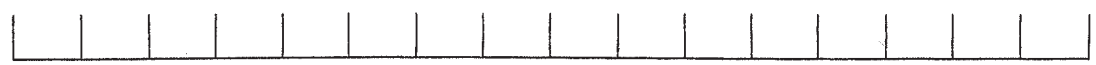

Date limite de validité : Mois, Année :

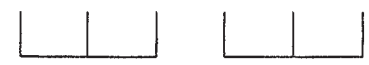

Signature obligatoire :

( ) Le règlement sera effectué à réception d'une facture, ou d'un mémoire administratif.

Date :

Signature : 


